\title{
Enhancing Flexibility of 2D Planar Materials By Applying Cut Patterns For Hands On Study Models
}

\author{
Orkan Zeynel Güzelci \\ Istanbul Kultur University, Turkey \\ orkanguzelci@gmail.com \\ Sema Alaçam \\ Istanbul Technical University, Turkey \\ semosphere@gmail.com
}

\author{
Zeynep Bacınoğlu \\ Istanbul Technical University, Turkey \\ zeynepbacinoglu@gmail.com
}

\begin{abstract}
Active experimentation during intertranslations between digital and physical modelling allow designers to explore new geometrical possibilities. Particularly, while changing the strength of the material, cut operations augment bending performance of the planar surfaces. Keeping in mind the potentiality of bending behavior as a generative tool for computational process, this paper presents the findings of three phased experimentation: implication of cut patterns to $2 \mathrm{D}$ planar material, mapping 2D patterns onto 3D surfaces and exploring new 3D free-form surfaces.
\end{abstract}

Keywords: Bending; Hands-on Exploration; Making; Laser Cut; Double Curvature

\section{Introduction}

Many designers and students consider design and manufacturing as separated phases. In other words, mostly production techniques are used in a precise and predefined way after the design conceptualization process finished. Today, there have been recent researches on Design Fabrication, which integrates material, form and performance aspects reciprocally, in which they inform each other. This integration is merging design and construction, designer and maker, which result with new material properties and unique fabrication techniques that can replace industrially standard materials and construction techniques.

In a broader sense, we propose to use a production technique as a design tool for integrating design and fabrication phases. In this study, production techniques of cutting and bending is adopted as a design tool. Cutting is used to create heterogeneous material properties for designing curvilinear surfaces through bending. Specifically, the study focuses of enriching the affordance of bending behavior of planar surface materials. With this in mind, we propose a three-phased model to integrate analog and digital design and modeling techniques. This model is based on applying cut patterns to $2 \mathrm{D}$ planar material, mapping $2 \mathrm{D}$ patterns onto $3 D$ surfaces and exploring new 3D free-form surfaces.

We began with physical explorations instead of starting with digital. Our model proposal allows discovering the advantages of hands-on experience to inform 3D digital models. We argue that it is crucial to understand potentiality of geometric surface patterning in relation with material properties beyond merely free form generation in the digital medium. It is because any of the relations learned from the material explorations would affect the whole fabrication process. Thus, the integration of analog and digital processes provide us to develop new possibilities for formal explorations informed by material properties.

The goals of this study are;

- To encourage usage of 2D available and cheap materials to construct 3D complex geometrical explorations as study model.

- To enhance the affordance of the planar material by using different cut operations.

- To gain experience from active experimentation during intertranslations between digital and physical modelling.

\section{Related Studies}

As Ackerman (2007) underlines in relation with Piaget's and Papert's discussions, making and construction are an important aspect of learning. In this sense, it is possible to consider digital fabrication techniques as an exploration space in which knowledge is constructed in the age of digital. In other words instead of using digital fabrication techniques precisely, we aim to construct design logic of fabrication based on learning from making experiments.

Integration of form generation techniques and material behavior is not a new issue in architecture. Apart from the precedent studies in ancient history, it is possible to trace back similar investigations in the 1930s in Bauhaus (MoholyNagy, 2005). In Moholy-Nagy's own words: "These woodcuttings show the potentialities of the woodworking machines until now unknown. Solid pieces of wood can be changed into rubber-like elasticity, etc. But what is more amazing is that same principle can be applied to every other material and every other tool."(Moholy-Nagy, 2005). 


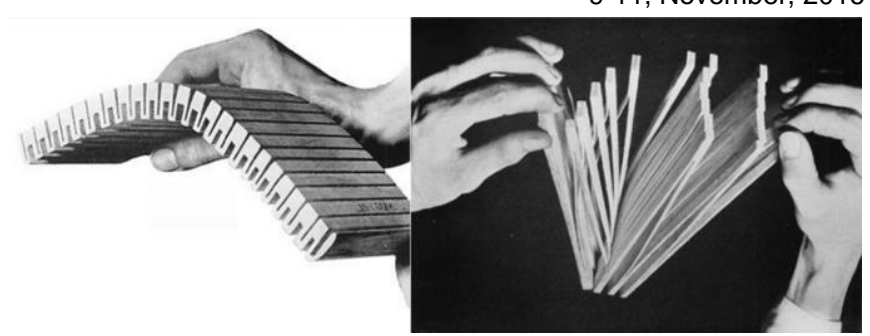

Figure 1: William Worst Woodcutting by Machine (left), Woodcutting by Bredendieck (right) in 1937(Moholy-Nagy, 2005, p.74-75)

As Carpo (2015) points, influenced by physical form finding methods of Frei Otto, Achim Menges can be considered as a pioneer in searching for forms by enriching the materials via computational implementations. According to Kotnik and Weinstock (2012), integrating form, material and force introduce a novel generative logic of form finding. As Kotnik and Weinstock (2012) state, material play an important role to make the form buildable. In many studies, the material is selected after finishing the conceptualization of form. However, we should emphasize that material, material behaviors, and fabrication techniques are important design aspects in the generation process. These design aspects are increasingly involved in contemporary design processes with the accessibility of digital technologies. For example, Weston (2012)'s experiments on unidirectional material weakening as a means of producing material properties for adjusting solar shading performance of building; Schönbrunner et al. (2015) uses cut patterns to transform the initial surface to ondulated surfaces for controlling the stiffness and self-supporting properties (2015); D'Acunto and Kotnik (2013) adopts cutting operation to control the bending resistance of the sheets for a larger spatial enclosure and reduced wind load acting upon the structure, and additionally produces a shadow pattern.

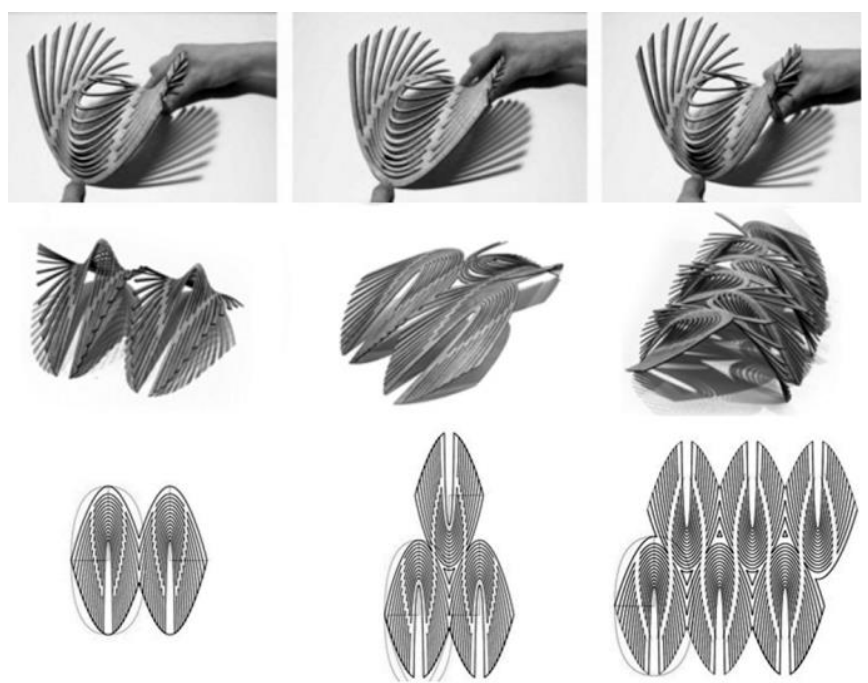

Figure 2: Self-weight bending behavior of plywood sheets, AA/ETH Pavilion (Url-1)

In addition to these, like in "The Wooden Waves" project illustrated in Figure 4, it is possible to claim that "folding" and "bending" operations are approached as a defined and complex engineering problem. In our study, we preferred to approach behavior of "bending" as an exploratory and playful process.
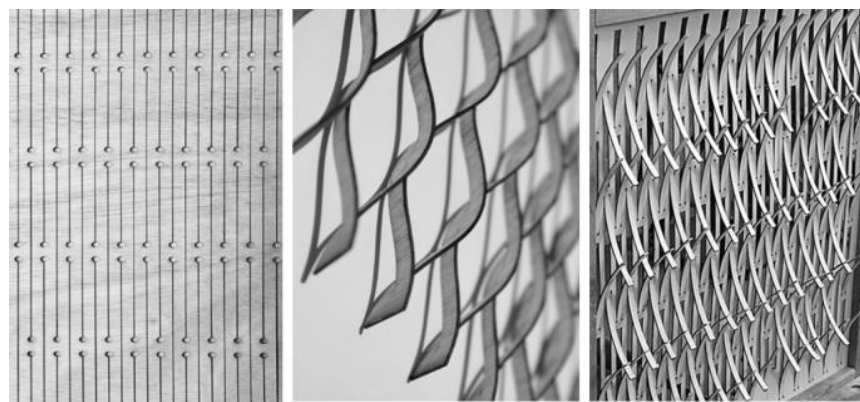

Figure 3: Unidirectional material weakening by Weston (2012).

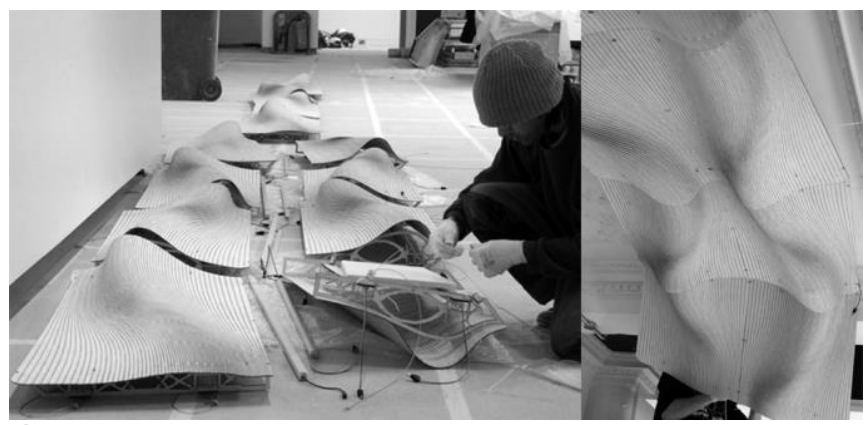

Figure 4: The Wooden Waves, by Mamou-Mani Architects in collaboration with BuroHappold Engineering (Url-2)

Outcomes produced during our experiments are study models rather than full-scale prototypes. Moreover, different than kerf cutting or engraving (via laser cut), we experimented with subtractive cut operations. In our study, planar wood materials are examined. The natural wood material has complex behaviors and anisotropic material properties. As we can benefit from natural anisotropic properties of natural (heterogeneous) materials; digital fabrication techniques can embed anisotropic properties into homogeneous materials. In this study, we used homogeneous planar board materials aiming to transform them to anisotropic wood-like material.

\section{Experiment Setup}

Medium density fiberboard (MDF), cardboard and plexiglas can be considered as common and available physical modeling materials. The scope of experimentation covers hands-on exploration, single material and implication of geometric surface patterning through operations of cut and bend. Rhinoceros/Grasshopper was used as a parametric modelling environment. Assumptions and variables of experiment setup are listed below:

- Size: The size of the planar cardboard material is limited as $10 \times 18 \mathrm{~cm}$.

- Thickness: The thicknesses of the materials are 1 and 2 millimeters.

- Pattern property: The four types (Figure 5) of the repetitive patterns applied to material via laser cutter 
- Level of porosity of the surface: The ratio of void area to solid area after the cutting operation.

- Bending behavior of the material: The maximum curvature of planar surface without being broken under the force of gravity and other external forces.

- Behavior: The possible formal reactions of the material to the bending operation during hands-on exploration from different pivot points.

- Translation: $d x$ and $d y$ are assumed to represent the distance between units in $X$ and $Y$ directions.

- Cut: Cut operations can be used to modify the porosity level of surface and flexibility of the material.

- Bend: Hands-on bend operations allow exploring new form possibilities and material properties.

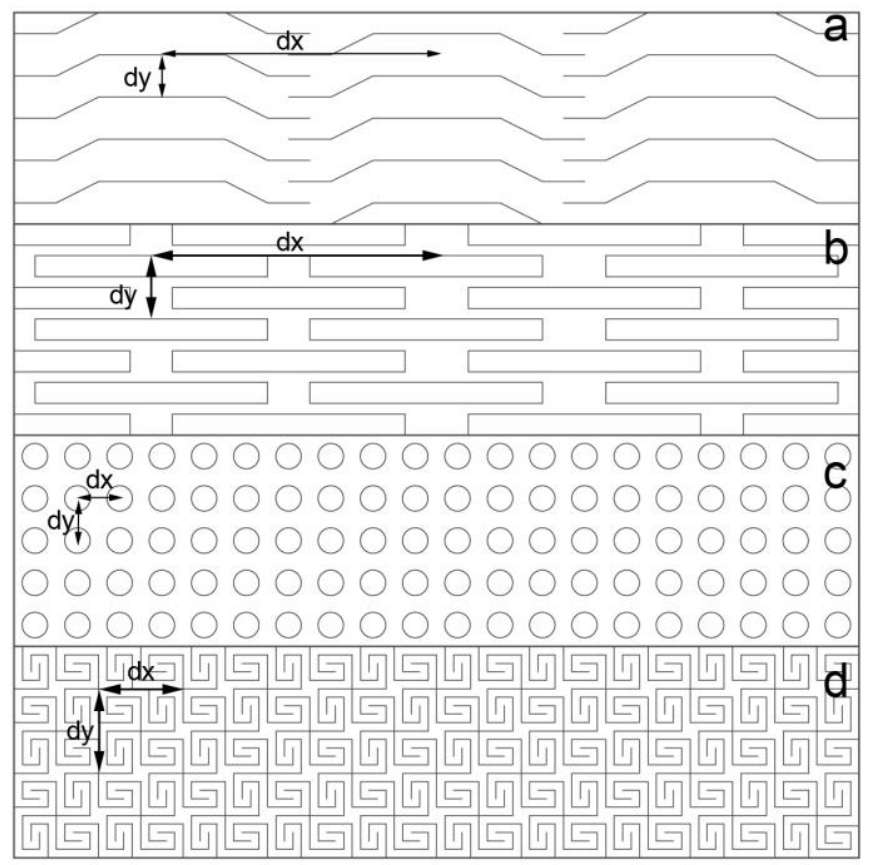

Figure 5: The selected four cut patterns

\section{Experimentation Process Observations}

The proposed model involves three phases: applying cut patterns to 2D planar material, mapping 2D patterns onto 3D surfaces and exploring new 3D free form surfaces.

The first phase is based on design of cut patterns and their implications onto 2D planar material via laser cut. Pattern design process starts with grid layout which has a predefined size. A single geometry is overlapped on intersection points of grid layout as a one or two dimensional array organization. Variables like size of the pattern units, distance and direction between pattern elements, thickness of the material are modified to explore the strength of the material. In first phase, the questions listed below were investigated:

- What is the relationship between pattern and the flexibility of the material?

- Are all isotropic materials behave in the same way?
- How can we come up with same 3D results with the materials with different thickness?

- What is the maximum bending limit of a material according to cut geometry?

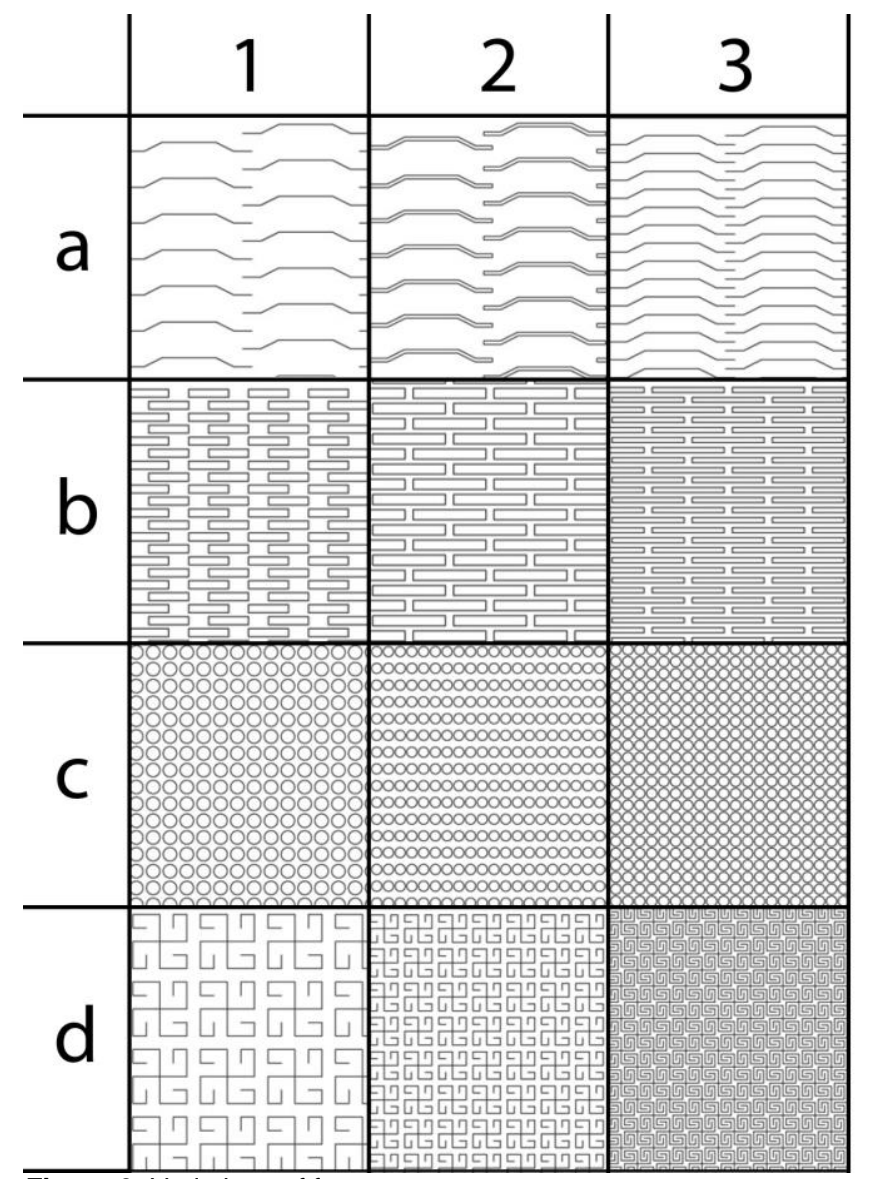

Figure 6: Variations of four cut patterns

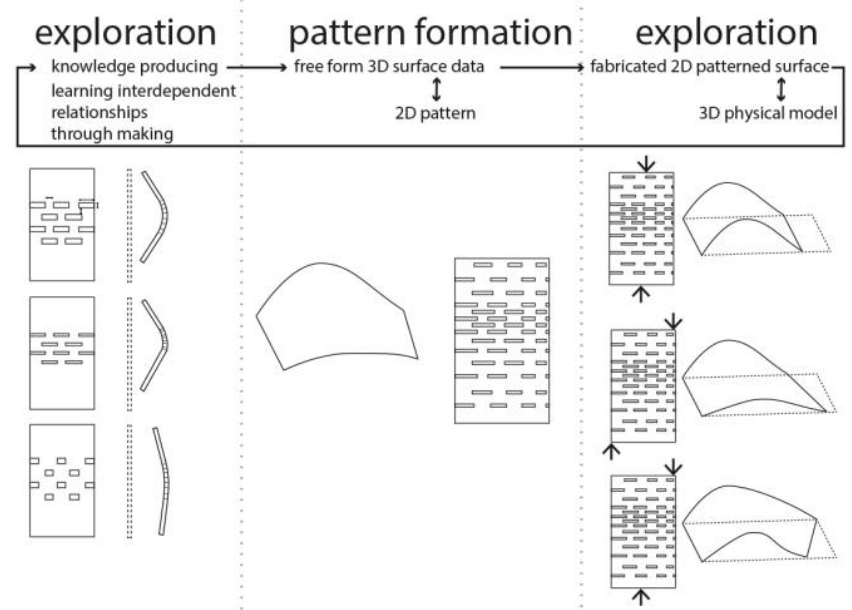

Figure 7: Model diagram.

One of the findings of this phase is that the porosity of the surface and the level of bending behaviors are not directly proportional. For example, in $f 4$ (Force directions of hands-on bending operations are shown in Figure 8 and Figure 9) voidsolid area ratio of $c 1$ surface is 1.01 (90.5 mm2 /89.5mm2) and bending angle of $c 1$ is 3 degree. The properties for $d 3$ 
are $1.23(99.5 \mathrm{~mm} 2 / 80.5 \mathrm{~mm} 2)$ and bending angle is 65 degree.
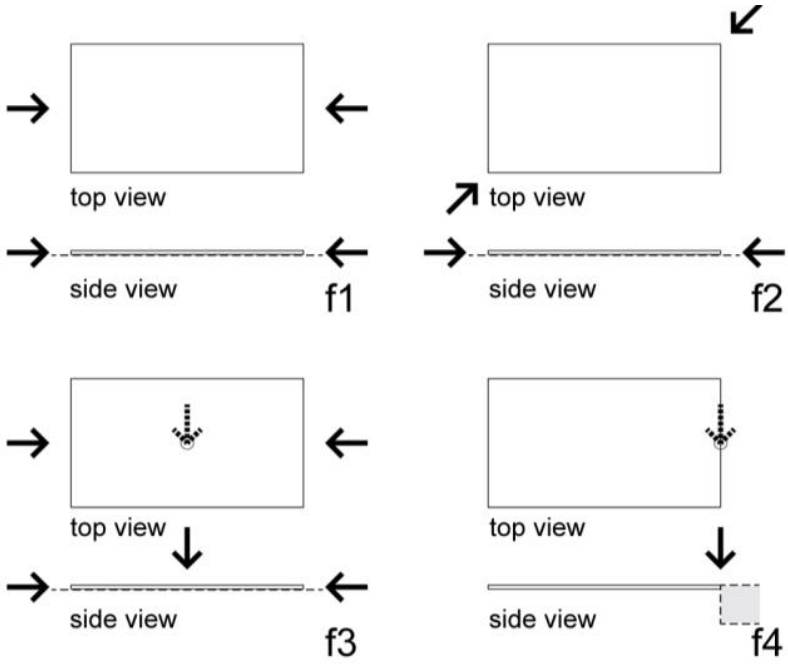

Figure 8: Operations and forces applied to the material.

This result shows us it is not the subtraction operation itself that increases the bending ability of the surface. But, the decreasing the strength of the surface especially in one direction through intertwining organization of the geometry might increase the flexibility of the material. In that way the planar surface begin to perform anisotropic behavior. The planar surface which performed the fabric-like maximum bending behavior was $d 3$ although the density of the pattern in both $\mathrm{X}$ and $\mathrm{Y}$ directions were the same.

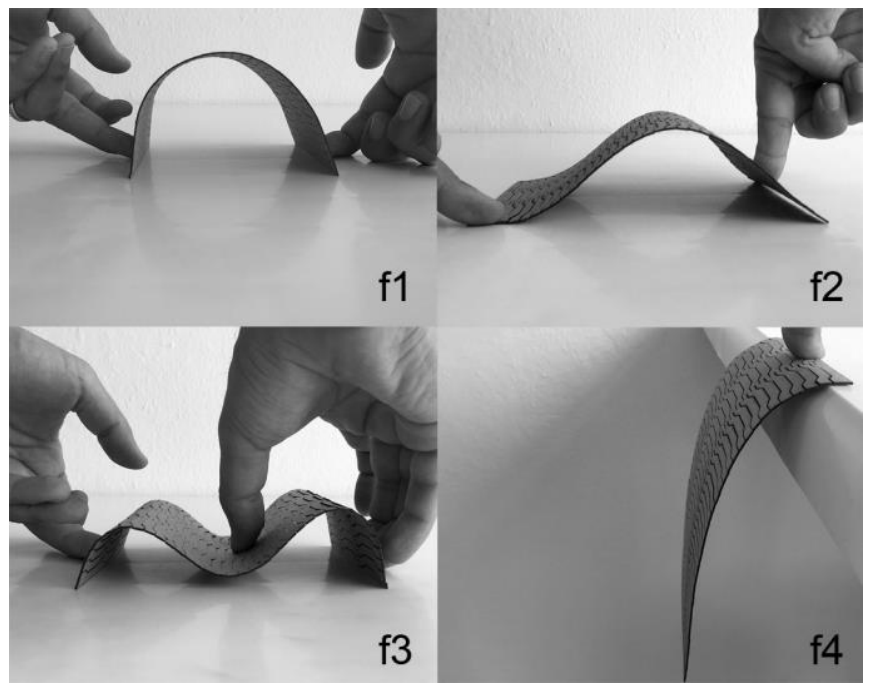

Figure 9: Photos from explorations of a3 pattern

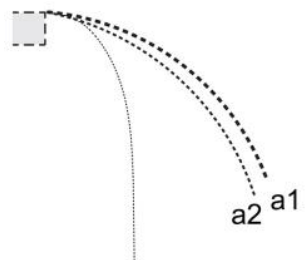

a3

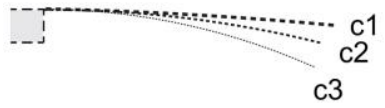

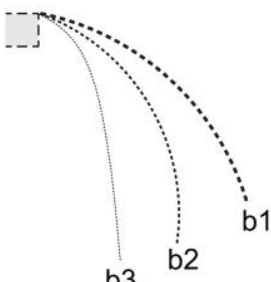

b3

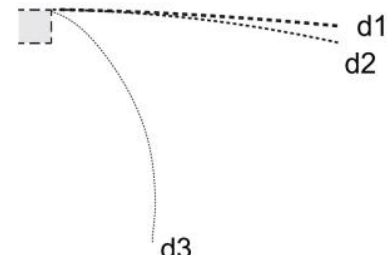

d3
Figure 10: Comparison of bend behavior in different pattern variations

After experimenting with $1 \mathrm{~mm}$ and $2 \mathrm{~mm}$ cardboard materials, we also discovered that the bending behavior and bending angle is related with thickness.

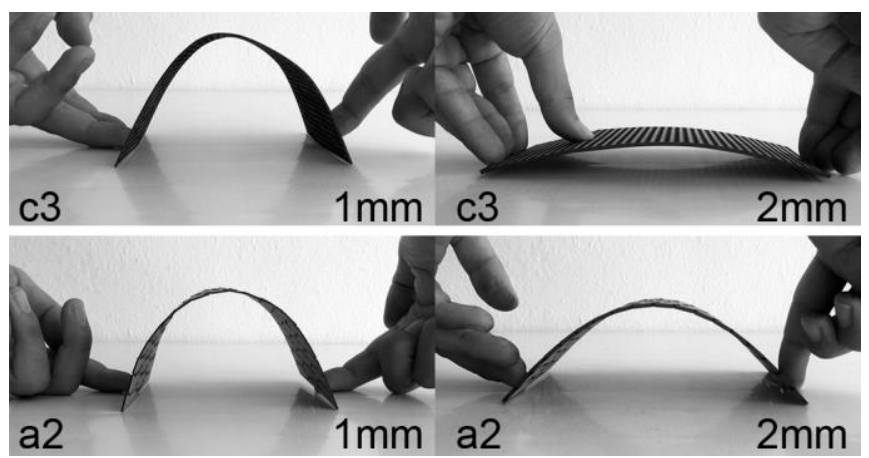

Figure 11: Same $f 1$ force applied to materials with different thickness

In some cases where hands-on experience is not used, bending behavior of a surface under $f 1$ and $f 2$ forces can be assumed as identical and symmetrical. In hands on exploration, hand movements cause deviations on symmetry axis (Figure 12). The obliquity in this modification is considered as an important parameter while transferring the information of analog model into computational model (Figure 13).

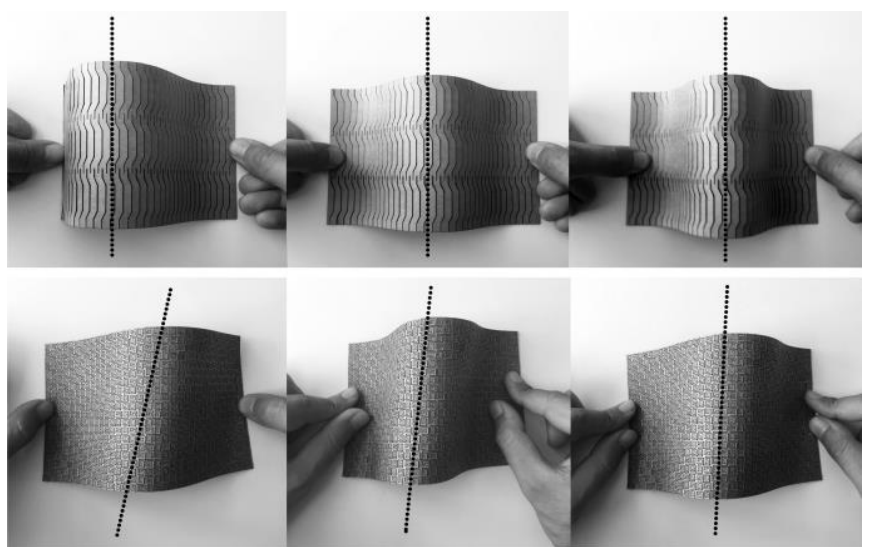

Figure 12: Obliquity on the symmetry axis under forces. 


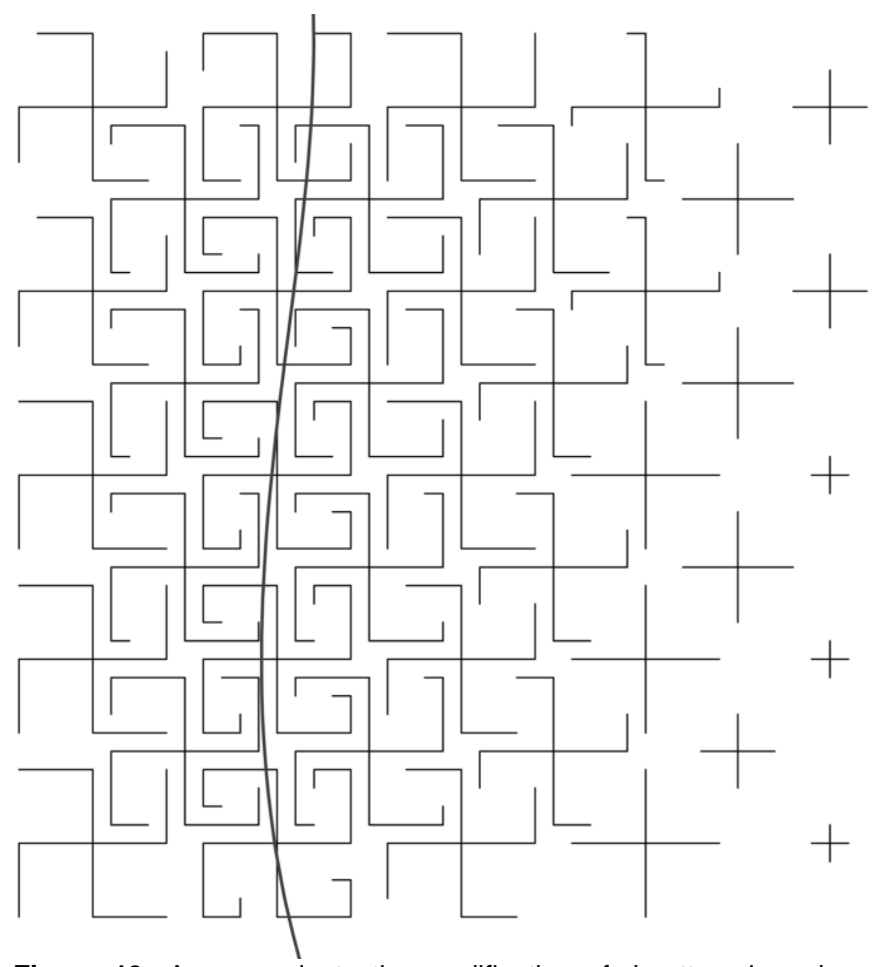

Figure 13: An example to the modification of d-pattern based on bending axes of the surface.

Understanding the relation between the properties of the geometric pattern and the properties of the material in an iterative way reveals computational logic that informs in the second phase. Among many trials, four patterns and their three variations were selected to exemplify these interdependent relations between geometric patterns and material property.

The second phase involves a series of reductions and new assumptions during the translation of curvature data into digital environment. Inputs of this phase are an initial 3D surface geometry with hills and valleys and also information derived from the first phase. The curvature on 3D surface and the parameters of $2 \mathrm{D}$ pattern are linked in $3 \mathrm{D}$ visual programming environment (Rhino/Grasshopper). According to the radius (curvature level) of the curves the center point of pattern units are colored. The white points refer to valleys with a lowest slope and the black points refer to hills or holes with a highest curvature level. In coding environment, number sliders are used to change the geometry and the scale of pattern elements (Figure 14). Afterwards, one of the selected patterns (Figure 6) is mapped onto planar surface in different parameters derived from the curvature data of 3D surfaces. The thresholds in the change of the curvature of the surface are used as attraction curves.

In other words, the curve connecting the inflection points on the $3 \mathrm{D}$ surface is projected onto the planar surface. This 2D curve is assumed as an attraction curve to modify the selected 2D patterns (Figure 13 and Figure 14).

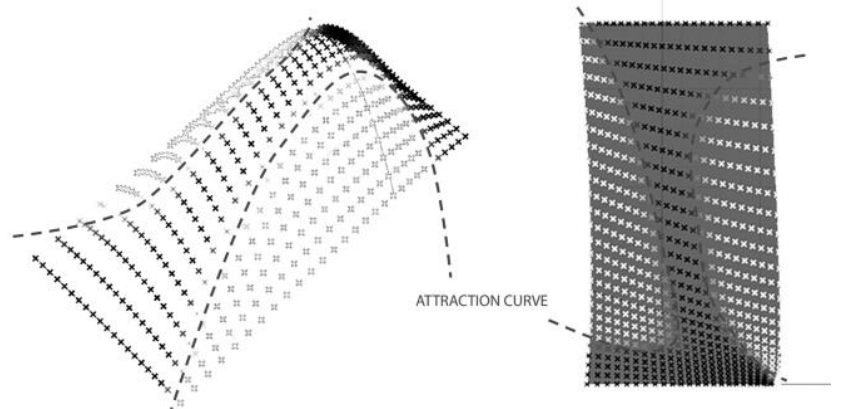

Figure 14: Sample to color coding of valley and hills
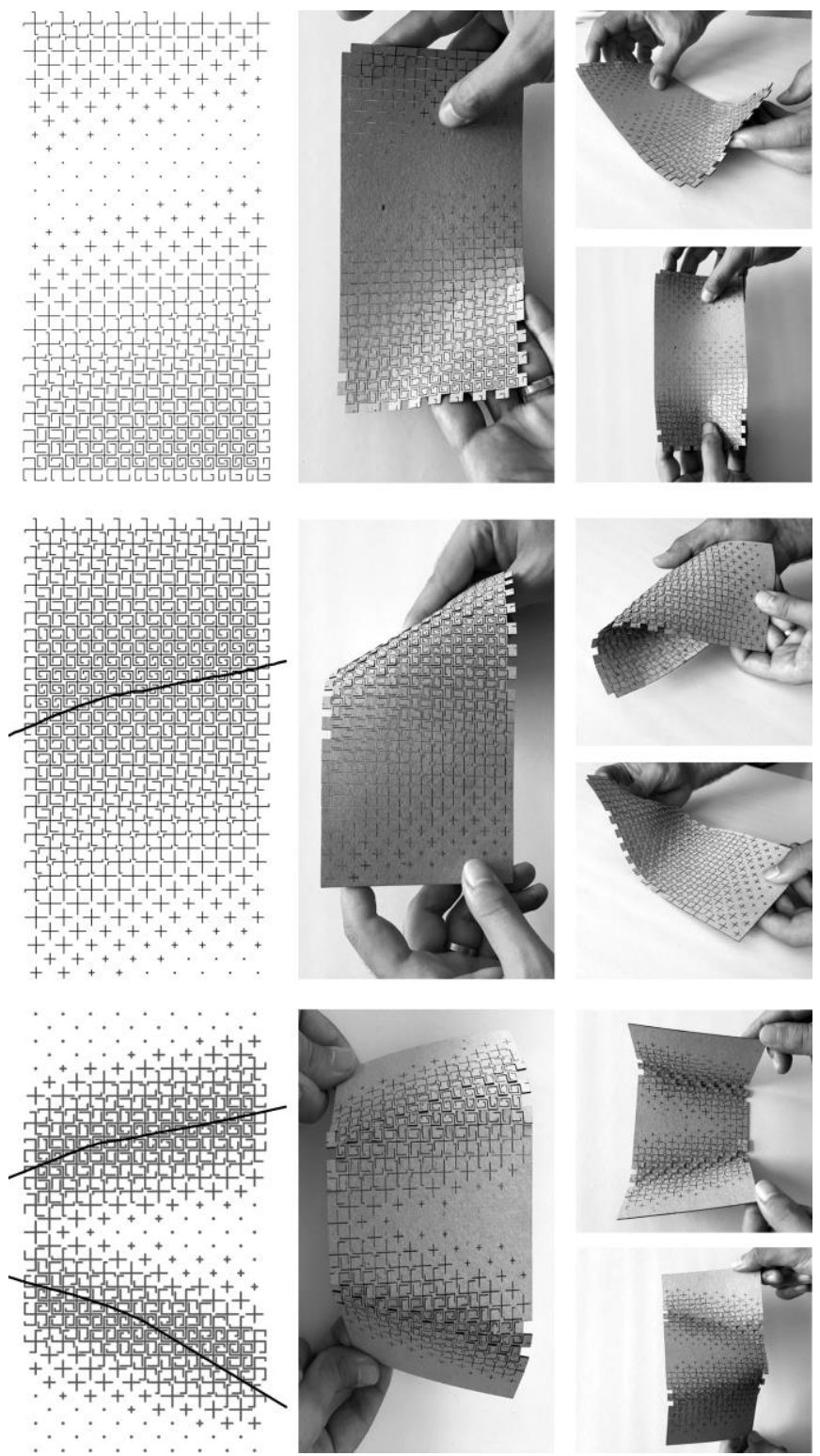

Figure 15: An example to the modification of d-pattern based on bending axes of the surface.

The third phase is hands-on explorations by using the laser cut productions in order to achieve various form possibilities. Outcomes of the second phase were used. Even, the 2D pattern is generated with the information derived from 3D 
model, hand-on exploration on 2D surface can generate new/various 3D surfaces (Figure 15).

\section{Discussion}

In this study, we focused on the question "How bending behavior become a generative tool for computational process?". With this interest we aimed to gain experience from active experimentation during cyclic explorations on bending operation in both digital and physical media.

Through exploring how properties and relations of twodimensional geometric patterns allows bending behavior, it is possible to gain an insight on the relation between $2 \mathrm{D}$ and 3D. Moreover, the model proposed in this study can be used as pedagogical approach which can encourage to use not only the material techniques of cutting and bending but also the other various techniques such as twisting, folding, kerfing, stretching in an explorative and creative way for integrative design formation and fabrication processes.

In this study, the observations and findings during the handson experimentation on 2D planar surface contributed reflective updates not only to digital model but also the organization of the geometry, 2D modifications of the cut pattern and the $3 \mathrm{D}$ explorations with the bending behavior.

Despite the fact that the material and the geometrical constraints of the experimentation were so limited, the response of the materials to the cut pattern operations have been different than our initial assumptions. The real-time material behaviors affect the whole digital modelling and fabrication process from the very beginning of the explorations.

In digital media we defined the surface geometry based on 2D array organizations and grid layout for pattern design. This grid layout itself became a limitation along the experiments. In the further studies the manipulations of the grid organization might enrich the geometric pattern generation process, thus the bending behavior.
The study process can be summarized as a research on enhancing the affordance of the planar material by using different cut operations through semi-structured experiments.

\section{Acknowledgments}

The authors would like to thank to İnanç Şencan for his contributions in the computational interpretations.

\section{References}

Ackeirmann, E. K. (2007). Experiences of Artifacts, Keyworks in radical constructivism. Ernst von Glasersfeld. M. Larochelle, (Ed.), (pp. 249-259). Rotterdam, Taipei: Sense Publishers.

Carpo, M. (2015). The New Science of Form-Searching. Architectural Design. 85(5): 22-27.

Kotnik, T., \& Weinstock, M. (2012). Material, form and force. Architectural Design, 82(2), 104-111.

Menges, A. (2011). Integrative design computation: Integrating material behaviour and robotic manufacturing processes in computational design for performative wood constructions. In Proceedings of the 31th Annual Conference of the Association for Computer Aided Design in Architecture (ACADIA), Banff (Alberta), pp. 72-81.

Moholy-Nagy, L. (2005). The New Vision: Fundamentals of Bauhaus Design, Painting, Sculpture, and Architecture, New York: Dover Publications.

D'Acunto, P., \& Kotnik, T. (2013) AA/ETH-Pavilion, Proceedings of the TENSINET Symposium 2013, Istanbul, 99-108

Schleicher, S., Rastetter, A., La Magna, R., Schönbrunner, A., Haberbosch, N., \& Knippers, J. (2015). Form-Finding and Design Potentials of Bending-Active Plate Structures. In Modelling Behaviour (pp. 53-63). Springer International Publishing.

Schönbrunner, A., Haberbosch, N., Magna, R. L., Schleicherab, S., Lienhard, J., \& Knippers, J. (2015) Design strategies for bendingactive plate structures out of multiple cross-connected layers. Proceedings of the International Association for Shell and Spatial Structures (IASS). Amsterdam, The Netherlands.

Weston, M. (2012). Anisotropic Operations. International Journal of Architectural Computing, 10(1), 105-120.

Url-1http://www.evolo.us/architecture/temporary-timber-pavilion/

Url-2 http://www.mamou-mani.com/burohappold/

Url-3 http://www.instructables.com/id/Super-flexible-duble-curvaturesurface-laser-cut- $p /$ 